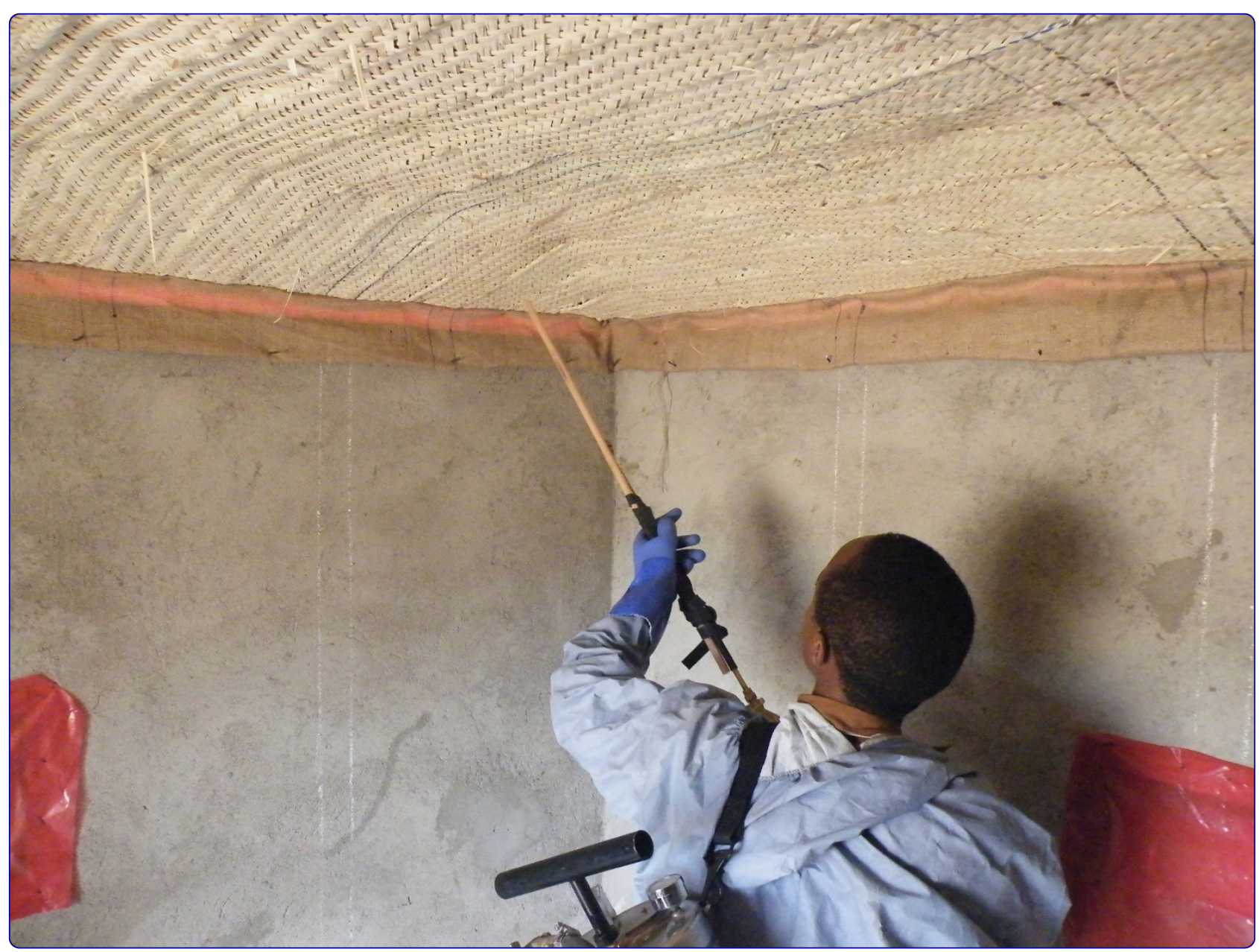

Long-lasting control of Anopheles arabiensis by a single spray application of micro-encapsulated pirimiphos-methyl (Actellic ${ }^{\circledR} 300$ CS)

Oxborough et al. 


\title{
Long-lasting control of Anopheles arabiensis by a single spray application of micro-encapsulated pirimiphos-methyl (Actellic ${ }^{\circledast} 300$ CS)
}

Richard M Oxborough ${ }^{1,2,3^{*}}$, Jovin Kitau ${ }^{2,3}$, Rebecca Jones ${ }^{1}$, Emmanuel Feston², Johnson Matowo ${ }^{2,3}$, Franklin W Mosha ${ }^{2,3}$ and Mark W Rowland ${ }^{1,3}$

\begin{abstract}
Background: Pyrethroid-resistant mosquitoes are an increasing threat to malaria vector control. The Global Plan for Insecticide Resistance Management (GPIRM) recommends rotation of non-pyrethroid insecticides for indoor residual spraying (IRS). The options from other classes are limited. The carbamate bendiocarb and the organophosphate pirimiphos-methyl ( $p$-methyl) emulsifiable concentrate (EC) have a short residual duration of action, resulting in increased costs due to multiple spray cycles, and user fatigue. Encapsulation (CS) technology was used to extend the residual performance of $\mathrm{p}$-methyl.
\end{abstract}

Methods: Two novel p-methyl CS formulations were evaluated alongside the existing EC in laboratory bioassays and experimental hut trials in Tanzania between 2008-2010. Bioassays were carried out monthly on sprayed substrates of mud, concrete, plywood, and palm thatch to assess residual activity. Experimental huts were used to assess efficacy against wild free-flying Anopheles arabiensis, in terms of insecticide-induced mortality and blood-feeding inhibition.

Results: In laboratory bioassays of An. arabiensis and Culex quinquefasciatus both CS formulations produced high rates of mortality for significantly longer than the EC formulation on all substrates. On mud, the best performing CS killed $>80 \%$ of An. arabiensis for five months and $>50 \%$ for eight months, compared with one and two months, respectively, for the EC. In monthly bioassays of experimental hut walls the EC was ineffective shortly after spraying, while the best CS formulation killed more than $80 \%$ of An. arabiensis for five months on mud, and seven months on concrete. In experimental huts both CS and EC formulations killed high proportions of free-flying wild An. arabiensis for up to 12 months after spraying. There was no significant difference between treatments. All treatments provided considerable personal protection, with blood-feeding inhibition ranging from 9-49\% over time.

Conclusions: The long residual performance of p-methyl CS was consistent in bioassays and experimental huts. The CS outperformed the EC in laboratory and hut bioassays but the EC longevity in huts was unexpected. Long-lasting p-methyl CS formulations should be more effective than both p-methyl EC and bendiocarb considering a single spray could be sufficient for annual malaria control. IRS with p-methyl 300 CS is a timely addition to the limited portfolio of long-lasting residual insecticides.

Keywords: IRS, Pirimiphos-methyl, Actellic, Anopheles arabiensis, Vector control, Malaria, Tanzania

\footnotetext{
* Correspondence: oxandbull@hotmail.com

'Department of Disease Control, London School of Hygiene and Tropical Medicine (LSHTM), London, UK

2Department of Entomology and Parasitology, Kilimanjaro Christian Medical University College (KCMUCo) of Tumaini University, Moshi, Kilimanjaro,

Tanzania

Full list of author information is available at the end of the article
}

\section{Ciomed Central}

(c) 2014 Oxborough et al.; licensee BioMed Central Ltd. This is an Open Access article distributed under the terms of the Creative Commons Attribution License (http://creativecommons.org/licenses/by/2.0), which permits unrestricted use, distribution, and reproduction in any medium, provided the original work is properly credited. The Creative Commons Public Domain Dedication waiver (http://creativecommons.org/publicdomain/zero/1.0/) applies to the data made available in this article, unless otherwise stated. 


\section{Background}

Indoor residual spraying (IRS) has produced profound changes in malaria burden in a range of settings with several different insecticide classes [1]. Interruption of malaria transmission in the USA was achieved partly through DDT house-spraying and led to the initiation of the World Health Organization (WHO)-led Global Malaria Eradication Scheme (1955-1969) [2]. Malaria was subsequently eradicated from Europe, parts of the Soviet Union, Israel, Lebanon, Syria, Japan, and Chinese Taiwan. Despite numerous positive outcomes, the benefits were not on the global scale that was anticipated. There were about 20 pilot IRS projects in sub-Saharan Africa between the mid 1950s and early 1960s [3] that demonstrated IRS significantly reduced malaria transmission even in highly endemic (intense transmission) areas [4]. Despite this, Africa was largely sidelined for eradication due to the high malaria burden; while elsewhere dramatic reversals were seen once IRS spraying was prematurely reduced in countries such as India and Sri Lanka [5,6]. As a result interest in IRS subsequently waned and was not taken to scale in most sub-Saharan malaria-endemic countries as part of the global eradication campaign [4,7].

Southern Africa was the exception. IRS programmes using DDT began in the 1960s and were supported for several decades, with later introduction of pyrethroids and carbamates. Countries with sustained IRS activities in Africa, including South Africa, Zambia, Namibia, Swaziland, Zimbabwe, and Botswana, achieved sizeable reductions in malaria vector populations and malaria incidence [7]. Focal IRS in the southern Africa region has remained important in areas of higher malaria burden and at risk of epidemics. In 2007, about 14 million people in southern Africa were protected by IRS $[4,7]$.

In $2006 \mathrm{WHO}$ reaffirmed the importance of IRS as a primary intervention for reducing or interrupting malaria transmission $[8,9]$. In recent years an unprecedented level of funding has initiated new IRS campaigns across sub-Saharan Africa, often in parallel with long-lasting, insecticide-treated bed nets (LLIN) distribution. In 2012 President's Malaria Initiative (PMI) supported IRS in 15 African countries, covering seven million structures [10]. The implementation of new IRS programmes, together with sustained IRS programmes in Southern Africa has elevated the importance of IRS as a primary intervention for malaria control in Africa. Greater emphasis has been placed on ensuring that IRS in Africa can be sustained [11].

Pyrethroids are the only group of insecticides approved by WHO Pesticide Evaluation Scheme (WHOPES) for LLINs [12]. Pyrethroid insecticides have also been preferred for IRS in Africa in recent years due to low cost, longevity of three to six months, and low mammalian and non-target toxicity [13]. Subsequently, pyrethroid resistance has become widespread in malaria vectors across Africa [14]. Reduced efficacy of insecticide interventions in areas of pyrethroid resistant malaria vectors has been demonstrated in several settings. A notable example is in South Africa where four years after the introduction of deltamethrin IRS a four-fold increase in malaria cases was recorded in Kwa-Zulu Natal, coinciding with re-invasion of pyrethroid resistant Anopheles funestus s.s. This trend was reversed after re-introduction of IRS with DDT in 2000 and new introduction of artemisinin-based combination therapy in 2001, with an accompanied decline in malaria cases by $91 \%$ [15]. In Bioko Island, Equatorial Guinea a single spray round with pyrethroid failed to reduce the population density of pyrethroid-resistant Anopheles gambiae s.s. Subsequent spraying of a carbamate significantly reduced the number of An. gambiae s.s. caught exiting in window traps, thus demonstrating the utility of non-pyrethroid IRS [16].

The residual lifespan of alternative IRS insecticides is of key importance. Based on WHOPES recommendation, DDT is the longest lasting IRS, with a duration of effective action greater than six months [17]. However, the Stockholm Convention on Persistent Organic Pollutants stipulates that, 'countries using DDT are encouraged to reduce and eliminate the use of DDT over time and switch to alternative insecticides' [18]. Carbamates are a commonly used alternative to DDT and pyrethroids, and were sprayed in ten African countries in 2012 through PMI funding. Based on WHOPES recommendation, bendiocarb has a short residual action of only two to six months [17]. In areas of intense yearround (perennial) transmission, multiple spray rounds of short lasting insecticides are expensive, logistically demanding, and inconvenient to householders [8]. Despite added impetus for the development of new public health insecticides, notably from Innovative Vector Control Consortium (IVCC), alternative classes of insecticide for public health use are emerging slowly [11]. For improved cost-effectiveness of IRS programmes it is important to develop new long-lasting formulations of currently available insecticides [19].

Encapsulation technology can extend the residual performance of established insecticides. Pirimiphos-methyl (p-methyl) is an organophosphate insecticide, most commonly and intensively used in the protection of cereal grain [20]. Several small and medium scale IRS trials conducted since the 1970s showed high toxicity to anopheline mosquitoes [21], leading to WHOPES' recommendation. According to WHOPES, p-methyl EC formulation has a relatively short residual IRS activity of two to three months but was used successfully for IRS in Malawi and Zambia in 2012 [22]. The overall aim of this study was to evaluate longevity of two capsule 
suspension (CS) formulations in comparison with emulsifiable concentrate (EC).

\section{Methods}

\section{Insecticide formulations}

Two capsule suspension (CS) formulation variants of Actellic 300CS, containing $300 \mathrm{~g} / \mathrm{L}$ p-methyl and coded as CS 'B' and CS 'BM' (Syngenta, Basel, Switzerland) were evaluated alongside the existing $\mathrm{EC}$ formulation (Actellic 50EC ${ }^{\oplus}$, Syngenta, Basel, Switzerland) in laboratory bioassays and experimental hut trials at $1 \mathrm{~g} / \mathrm{m}^{2}$. Lambdacyhalothrin CS $\left(0.03 \mathrm{~g} / \mathrm{m}^{2}\right)$ (Icon $\mathrm{CS}^{\bullet}$, Syngenta, Basel, Switzerland) is a WHOPES recommended formulation that was sprayed in Tanzania as part of the national malaria control programme (NMCP) from 2007-2012 [23] and was included in laboratory bioassays as a positive control but was not sprayed in experimental huts (due to availability of huts).

\section{Laboratory assessment of residual performance}

Cone bioassays to assess insecticidal duration on sprayed mud, concrete and plywood substrates were conducted every month based on WHO guidelines [9]. Substrates were stored at ambient temperature and humidity ( 20$28^{\circ} \mathrm{C}, 40-80 \% \mathrm{RH}$ ). For each formulation three blocks were sprayed and $\sim$ nine replicates of $\sim$ ten female Anopheles arabiensis were tested, (i.e. three replicates per block), for an exposure of 60 minutes. This is longer than the $30 \mathrm{mi}-$ nutes standard exposure time as specified by WHO for IRS cone bioassay, regardless of the insecticide [9]. Test mosquitoes were transferred to $150 \mathrm{ml}$ paper cups with $10 \%$ glucose solution provided ad libitum and mortality recorded after 24 hours. Substrates were sprayed at an application rate of $40 \mathrm{ml} / \mathrm{sq} \mathrm{m}$ using a Potter Tower Precision Sprayer (Burkard Scientific, Uxbridge, UK). Resistance status of insectary-reared female test mosquitoes $A n$. arabiensis Dondotha, Culex quinquefasciatus TPRI and $C x$. quinquefascaistus Muheza was determined in WHO susceptibility tests (Table 1 ).

\section{Indoor residual spraying experimental hut trials}

An experimental hut trial was conducted at Kilimanjaro Christian Medical University College (KCMUCo) Field Station in Lower Moshi Rice Irrigation Zone $\left(3^{\circ} 22^{\prime} \mathrm{S}, 37^{\circ}\right.$ $\left.19^{\prime} \mathrm{E}\right)$ nightly for 12 months between December 2008 and December 2009. The walls and ceiling of the p-methyl EC hut were covered with untreated plastic sheeting for 1 month in January 2010 to investigate the possibility of mosquito movement between huts. To determine the relative contribution of the sprayed mud and concrete walls to mortality of $A n$. arabiensis the palm thatch ceiling was covered with unsprayed plastic sheeting every second week for 2 months from March-April 2010 in all huts. Further description of the supplementary experimental hut tests is included in the results section. Anopheles arabiensis densities were heavily dependent on rice cropping cycles with flooded rice fields adjacent to the Field Station being the main breeding site. In 2009, wild An. arabiensis were tested in WHO cylinder bioassays and were found to be susceptible to organophosphates, including p-methyl, and resistant to permethrin (Table 2).

Verandah experimental huts were constructed to a design described by WHO [9]. The working principle of these huts has been described previously [24]. The interior walls of experimental huts were plastered with either mud or concrete. A palm thatched mat, typical of organic fibres used in some rural housing [25], was affixed to the wooden ceiling before spraying.

The walls and ceiling were sprayed at an application rate of $40 \mathrm{ml} / \mathrm{sq} \mathrm{m}$ with a Hudson X-pert sprayer (H D Hudson Manufacturing Company, Chicago, Ill, USA) with flat fan $8002 E$ nozzle [26]. A constant flow valve (CFV) was not used, but compression was maintained at 55 psi by repressurizing after each swath. Flow rate was $840 \mathrm{ml} /$ minute. A guidance pole was used to ensure a consistent vertical swath $71 \mathrm{~cm}$ wide and swath boundaries were marked out with chalk on walls and ceiling to improve spray accuracy. High performance liquid chromatography (HPLC) was not done to confirm the accuracy of the spray concentration. Verandahs were protected during spraying by blocking the open eaves with a double layer of plastic and Hessian sackcloth. IRS treatments were randomly assigned to huts. Rotation of IRS treatments was not feasible as the mud and concrete substrates were permanent. Hut position is known to bias the number of mosquitoes entering a hut, but is unlikely to affect the primary proportional outcomes, per cent mortality and per cent blood-fed of

Table 1 Resistance status of insectary-reared mosquitoes to pyrethroid and organophosphate insecticides

\begin{tabular}{lccc}
\hline \multicolumn{1}{c}{ Species } & Strain & \% Mortality (n) & Malathion 5\% \\
\hline Anopheles arabiensis & Dondotha & $100(100)$ & $100(100)$ \\
Culex quinquefasciatus & TPRI & $97(208)$ & $99(200)$ \\
Culex quinquefasciatus & Muheza & $35(105)$ & $100(200)$ \\
\hline
\end{tabular}

Results of susceptibility testing with insectary strains exposed for one hour using WHO diagnostic dosages in cylinder bioassays. 
Table 2 Resistance status of wild Anopheles arabiensis to pyrethroid and organophosphate insecticides

\begin{tabular}{cccc}
\hline Insecticide & Dosage (\%) & Number tested & Mortality (\%) \\
\hline P-methyl & 0.025 & 40 & 98 \\
P-methyl & 0.05 & 40 & 100 \\
P-methyl & 0.25 & 40 & 100 \\
Malathion & 5 & 201 & 100 \\
Permethrin & 0.75 & 111 & 90 \\
\hline
\end{tabular}

Two- to five-day old sugar-fed offspring (F1) of Anopheles arabiensis collected from cattle-sheds in Lower Moshi were exposed for one hour in WHO cylinders lined with papers treated with diagnostic dosages of malathion and permethrin, and a range of dosages of p-methyl.

those entering the huts. The following treatments were sprayed in a total of six experimental huts.

- Pirimiphos methyl CS 'B', 1 g/sq m (one mud and one concrete walled hut)

- Pirimiphos methyl CS 'BM', $1 \mathrm{~g} / \mathrm{sq}$ m (one mud and one concrete walled hut)

- Pirimiphos methyl EC, $1 \mathrm{~g} / \mathrm{sq} \mathrm{m}$ (one mud walled hut)

- Unsprayed (one mud walled hut)

The trial protocols were based on WHOPES procedures for small-scale field trials for IRS [9]. Adult trial participants gave informed consent and were offered free medical services during the trial and up to three weeks after the end of participation. An adult volunteer slept in each hut nightly from 20:30-06:30. Sleepers were rotated between huts on successive nights to reduce any bias due to differences in individual attractiveness to mosquitoes. Each morning mosquitoes were collected from the verandahs and window traps of huts and recorded as blood-fed or unfed and dead or alive. Live mosquitoes in the sprayed room were not collected in order to allow for natural resting times on treated surfaces, and were only collected after exiting to verandahs or window traps. $10 \%$ glucose pads were placed in the window traps and verandahs to prevent death by starvation. Live mosquitoes were transferred to $150 \mathrm{ml}$ paper cups and provided with $10 \%$ glucose solution before scoring delayed mortality after 24 hours. All members of the An. gambiae species complex identified by morphological characteristics were assumed to be $A n$. arabiensis based on recent PCR identification [27].

\section{Experimental hut bioassays}

Cone bioassays of the sprayed walls and ceiling were conducted monthly using sugar-fed, two to five day-old female An. arabiensis dondotha, for an exposure of 60 minutes. In each experimental hut four to eight replicates of ten female mosquitoes were tested on the walls and ceiling surfaces. Cones were positioned randomly for each test.

\section{Fumigant activity}

The possibility of fumigant activity of the treatments was determined using insectary-reared wild female F1 An. arabiensis (no tarsal contact) [9]. Wire cages measuring $15 \mathrm{~cm} \times 10 \mathrm{~cm} \times 10 \mathrm{~cm}$ covered with netting were hung in the corner of the room $\sim 5 \mathrm{~cm}$ from the wall and 25 mosquitoes exposed overnight. Testing was done monthly in for all treatments until mortality decreased to low levels.

\section{Analysis of laboratory assessment of residual performance}

Treatments were compared according to the time interval since spray application for mortality to fall to $80 \%$ (based on WHOPES criteria) and 50\% [9]. Mixed effect logistic regression models were used to fit mortality trajectories over time separately for each strain of mosquito (An. arabiensis Dondotha, Cx. quinquefasciatus TPRI and $C x$. quinquefasciatus Muheza), treatment (P-methyl $\mathrm{EC}, \mathrm{CS}$ 'B' and CS 'BM' and lambdacyhalothrin CS) and substrate (mud, concrete and plywood). All statistical modelling was performed on the log odds scale at the individual mosquito level and results back transformed to the proportion scale. Linear, quadratic and cubic terms in time were specified as predictors in the models to allow for potential drops and then levelling off in mortality rates over time. A random effect was specified in all models to account for similarities in mosquitoes tested at the same time point and for potential behavioural clustering within the same test batch. The cubic equations given by the estimates from the polynomial models were solved to obtain estimates of the time points at which mortality fell to 80 and $50 \%$. Ninetyfive per cent confidence intervals $(\mathrm{CI})$ were estimated using the bias corrected bootstrap method with 2,000 replications. Differences between treatments in estimated time for mortality to fall to 80 and $50 \%$ were calculated and statistically significant differences inferred from the bootstrap $95 \%$ CI $(\mathrm{p}=0.05)$.

\section{Analysis of experimental hut bioassays}

Analysis of hut bioassays was similar to that described for laboratory bioassays. For wall assays, separate models were fitted for each hut. For ceiling assays, data from huts treated with the same insecticide (but with different wall materials) were combined. There was little evidence of a departure from a linear decrease in the log odds of mortality over time for either the wall or ceiling assays, so a linear term in time was specified as the only predictor in all models. 


\section{Analysis of experimental hut trial}

The number of mosquitoes collected from the two closed verandahs was multiplied by two to adjust for the unrecorded escapes through the two open verandahs which were left unscreened to allow routes for entry of wild mosquitoes via the gaps under the eaves $[9,24]$. The data were analysed to show the effect of each treatment in terms of:

Overall mortality $=$ Total proportion of mosquitoes dead on the morning of collection, plus delayed mortality after holding for a total of 24 hours; Blood feeding inhibition = Percentage of blood-fed mosquitoes from a treated hut relative to percentage from the unsprayed negative control;

Mortality-feeding index = The null hypothesis was that mortality and blood-feeding are independent so that mosquitoes surviving or killed by the treatment have an equal probability of having fed or not. Deviation from the null hypothesis shows whether there is association between feeding and mortality and may indicate the sequence of events experienced by individual mosquitoes after entering in the hut. The mortality-feeding index is calculated as follows:

$$
\begin{aligned}
& \text { Mortality-feeding index }= \\
& \text { (total blood-fed dead/total blood-fed) } \\
& \text {-(total unfed dead/total unfed)Interpretation } \\
& \text { of mortality-feeding index } \\
& 0=\text { equal chance of unfed and blood } \\
& \text {-fed mosquitoes being killed } \\
& 0 \text { to }-1=\text { deviation towards unfed mosquitoes } \\
& \text { being killed } \\
& 0 \text { to } 1=\text { deviation towards blood-fed mosquitoes } \\
& \text { being killed }
\end{aligned}
$$

Separate mixed effect logistic regression models were fitted to the mortality and blood-feeding data. The main predictors in each model were treatment, one or more time parameters and interactions between treatment and each of the time terms. There was little evidence of a departure from a linear decrease in the log odds of mortality over time since spraying, so only linear terms in time were specified in the statistical model for mortality. A model with linear, quadratic and cubic terms in time provided the best fit to the blood-feeding data. A random effect was specified in both models to account for similarities among mosquitoes entering huts on the same day and potential behavioural clustering. Both models controlled for sleeper. Predicted trajectories were plotted over the duration of the 12 months for mortality alongside actual results.

\section{Results}

\section{Laboratory residual bioassay}

The duration of residual activity of the p-methyl formulations on mud, concrete, and plywood are presented in Table 3 and the differences in residual activity are shown in Table 4 . Using $>80 \%$ mortality and $>50 \%$ mortality as the duration of residual efficacy, there was evidence that the two CS formulations showed significantly longer activity than the EC on mud and concrete substrates for both An. arabiensis and for two strains of $C x$. quinquefasciatus, but differences between the two CS formulations were non-significant in most instances. There was no evidence that treatment performance differed between species or strains.

When sprayed on mud, the EC had a particularly short residual action against An. arabiensis, and killed $>80 \%$ for only one month (95\% CI: 0.7-1.8). CS 'B' and CS 'BM' showed substantial improvement over the EC with mortality $>80 \%$ for 4.9 months (95\% CI: 4.4-5.5) and 4.4 months (95\% CI: 3.8-5.1) respectively $(\mathrm{P}<0.05)$. The residual times for $50 \%$ mortality to be reached, (RT 50 ), were 7.5 months ( $95 \%$ CI: 5.7 to $\dagger$ ) for CS 'B'; 6.2 months (95\% CI: 5.4-7.0) for CS 'BM'; and 1.9 months (95\% CI: 1.2-4.2) for EC (Table 3, Figure 1). On concrete CS ' $\mathrm{B}$ ' produced $>80 \%$ mortality for 4.1 months (95\% CI: 3.64.7) longer than the EC against An. arabiensis $(\mathrm{P}<0.05)$ (Table 4). Based on observed data on plywood, both CS 'B' and CS 'BM' killed $>80 \%$ An. arabiensis for 12 months. The EC killed $>80 \%$ for eight months, followed by a rapid decline to $<30 \%$ after nine months (Figure 2).

\section{Residual activity of formulations in experimental huts}

One-hour cone bioassays of An. arabiensis were conducted on walls and ceilings at monthly intervals. Both CS formulations showed improvement over the EC on mud, concrete and palm thatch. Mortality was $100 \%$ one week after spraying the CS 'B' and CS 'BM' formulations on mud and concrete walls (Figure 3). Mortality was $>80 \%$ for CS 'B' for 4.8 months (95\% CI: 1.9-6.9) on mud and 7.0 months (95\% CI: 5.4-8.3) on concrete, compared with 0.9 months (95\% CI: 0-4.4) and 6.6 months (95\% CI: 3.09.0) for CS 'BM' respectively (Table 5). The EC was ineffective on mud and killed a small proportion one week after spraying.

Actellic CS on palm thatch ceiling was highly effective, with close to $100 \%$ mortality recorded for both CS formulations after six months (Figure 4 ) and $>80 \%$ for 8.4 months for CS 'B' (95\% CI: 7.4-9.4) and 10.8 months for CS 'BM' (95\% CI: 9.9-11.7) (Table 5). Mortality remained high for the CS formulations and was $>50 \%$ up to 12 months (95\% CI: 11.2-12.7) and 14.4 (13.7-15.2) months after spraying for CS 'B' and 'BM' respectively. The EC initially killed a fairly high proportion of $A n$. arabiensis but showed a 
Table 3 Estimated time (months) for mortality to decrease to 80 and $\mathbf{5 0 \%}$ for Anopheles arabiensis, Culex quinquefasciatus TPRI and Muheza strains tested on laboratory sprayed substrates

\begin{tabular}{|c|c|c|c|c|c|}
\hline \multirow[t]{2}{*}{ Substrate } & \multirow[t]{2}{*}{ Insecticide } & \multicolumn{2}{|c|}{ Estimated time to $80 \%$ mortality } & \multicolumn{2}{|c|}{ Estimated time to $\mathbf{5 0} \%$ mortality } \\
\hline & & Time (months) & $95 \% \mathrm{Cl}$ & Time (months) & $95 \% \mathrm{Cl}$ \\
\hline \multicolumn{6}{|c|}{ Anopheles arabiensis dondotha } \\
\hline \multirow[t]{3}{*}{ Mud } & P-methyl EC & 1.0 & (0.7 to 1.8$)$ & 1.9 & $(1.2$ to 4.2$)$ \\
\hline & P-methyl CS B & 4.9 & (4.4 to 5.5$)$ & 7.5 & $(5.7$ to $\dagger)$ \\
\hline & P-methyl CS BM & 4.4 & (3.8 to 5.1 ) & 6.2 & (5.4 to 7.0$)$ \\
\hline \multirow[t]{3}{*}{ Concrete } & P-methyl EC & 2.3 & (1.8 to 2.7$)$ & 3.1 & (2.7 to 3.3$)$ \\
\hline & P-methyl CS B & 6.4 & (6.1 to 6.8$)$ & 7.2 & (6.9 to 7.5$)$ \\
\hline & P-methyl CS BM & 5.0 & (4.4 to 5.5 ) & 6.5 & (6.0 to 7.0$)$ \\
\hline \multicolumn{6}{|c|}{ Culex quinquefasciatus TPRI } \\
\hline \multirow[t]{4}{*}{ Mud } & P-methyl EC & 1.8 & (1.4 to 2.2$)$ & 2.1 & (1.7 to 2.5$)$ \\
\hline & Lambda CS & 2.9 & (2.7 to 3.3 ) & 3.7 & (3.4 to 4.0$)$ \\
\hline & P-methyl CS B & 6.2 & (5.3 to 7.6$)$ & t & t \\
\hline & P-methyl CS BM & 7.4 & (6.8 to 8.1$)$ & 9.7 & (8.6 to 11.0$)$ \\
\hline \multirow[t]{4}{*}{ Concrete } & P-methyl EC & 0.8 & (0.7 to 0.9 ) & 1.3 & $(1.2$ to 1.6$)$ \\
\hline & Lambda CS & 5.0 & (4.7 to 5.3$)$ & 5.9 & (5.7 to 6.1$)$ \\
\hline & P-methyl CS B & 8.2 & (7.5 to 9.3$)$ & 9.7 & (8.9 to 10.7$)$ \\
\hline & P-methyl CS BM & 6.8 & (0.6 to 7.2$)$ & 8.6 & (8.1 to 9.1$)$ \\
\hline \multicolumn{6}{|c|}{ Culex quinquefasciatus Muheza } \\
\hline \multirow[t]{4}{*}{ Mud } & P-methyl EC & 0.8 & (0.5 to 1.1$)$ & 1.3 & (1.0 to 1.6$)$ \\
\hline & Lambda CS & $\dagger$ & $\dagger$ & 0.9 & (0.5 to 1.4$)$ \\
\hline & P-methyl CS B & 4.0 & (3.5 to 4.6$)$ & 7.1 & (5.5 to 11.0$)$ \\
\hline & P-methyl CS BM & 3.8 & (3.3 to 4.3 ) & 6.4 & (5.7 to 7.3 ) \\
\hline \multirow[t]{4}{*}{ Concrete } & P-methyl EC & 1.0 & (0.8 to 1.2$)$ & 1.4 & $(1.0$ to 1.7$)$ \\
\hline & Lambda CS & 1.1 & (0.8 to 1.6$)$ & 1.8 & (1.5 to 2.2$)$ \\
\hline & P-methyl CS B & 4.9 & (4.2 to 5.6$)$ & 6.5 & (5.8 to 7.4$)$ \\
\hline & P-methyl CS BM & 4.3 & (4.1 to 4.6 ) & 5.7 & (5.3 to 6.1 ) \\
\hline
\end{tabular}

tIndicates that statistical models produced estimates outside the study period: for Culex quinquefasciatus TPRI, estimated mortality for Actellic CS-B on mud was higher than $50 \%$ throughout the entire study period; for Culex quinquefasciatus Muheza, estimated mortality for Lambda CS was lower than $80 \%$ throughout.

marked reduction to $<50 \% 2.4$ months (95\% CI: $0-6.1$ ) after spraying.

\section{Twelve-months experimental hut trial against wild}

\section{free-flying Anopheles arabiensis}

All formulations of p-methyl (CS 'B', CS 'BM', and EC) were highly effective against free-flying wild $A n$. arabiensis shortly after spray application (Figure 5). Mortality gradually decreased over time for all formulations up to five months after spraying, followed by a small increase between months five to seven, possibly due to climatic changes. Subsequently, between months seven to 12 there was a gradual decrease in mortality (Figure 5). Overall mortality rates remained high for both CS treatments up to12 months after spraying regardless of wall substrate. P-methyl EC performed equally well as CS 'B' and CS 'BM' after 12 months, based on 95\% CIs from estimated curves. Twelve months after spraying predicted mortality was $62.8 \%$ (95\% CI: 54.4-71.2) for EC, $72.0 \%$ (95\% CI: 64.5-79.6) for CS 'B' (mud) and 69.5\% (95\% CI: 62.0-77.0) for CS 'BM' (mud) (Table 6).

Blood-feeding was high in the unsprayed hut throughout the study but did show considerable variation over time and ranged from $40 \%$ (after nine months) to $90 \%$ (five and 12 months) (Figure 6). The two periods of lowest percentage blood feeding in the unsprayed hut, one and nine months after spraying, coincided with the period of highest mosquito density during rice transplantation cycles (Figure 6). For the first month after spraying, treated huts provided no protection from being bitten by hostseeking $A n$. arabiensis. Between two and 12 months after spraying all treatments provided some degree of personal protection (Figure 6). Blood-feeding inhibition was relatively high after six and nine months across all treatments 
Table 4 Between treatment differences in estimated time for mortality to fall to 80 and $50 \%$ for mosquitoes tested on insecticide-treated substrates

\begin{tabular}{|c|c|c|c|c|c|c|c|}
\hline \multirow[t]{2}{*}{ Substrate } & \multirow{2}{*}{$\begin{array}{l}\text { Treatment } \\
\text { comparison }\end{array}$} & \multicolumn{3}{|c|}{ Difference in estimated time to $80 \%$ mortality } & \multicolumn{3}{|c|}{ Difference in estimated time to $50 \%$ mortality } \\
\hline & & Time months & $95 \% \mathrm{Cl}$ & $\mathrm{p}$ & Time months & $95 \% \mathrm{Cl}$ & $\mathrm{p}$ \\
\hline \multicolumn{8}{|c|}{ Anopheles arabiensis dondotha } \\
\hline \multirow[t]{3}{*}{ Mud } & CS B vs EC & 3.9 & (3.1 to 4.6$)$ & $<0.05$ & 5.6 & (3.0 to 12.9$)$ & $<0.05$ \\
\hline & CS BM vs EC & 3.5 & (2.6 to 4.3 ) & $<0.05$ & 4.2 & $(2.0$ to 5.4$)$ & $<0.05$ \\
\hline & CS B vs CS BM & 0.4 & $(-0.4$ to 1.3$)$ & $n / s$ & 1.3 & $(-0.7$ to 11.7$)$ & $\mathrm{n} / \mathrm{s}$ \\
\hline \multirow[t]{3}{*}{ Concrete } & CS B vs EC & 4.1 & (3.6 to 4.7 ) & $<0.05$ & 4.1 & (3.7 to 4.6$)$ & $<0.05$ \\
\hline & CS BM vs EC & 2.6 & (1.9 to 3.4$)$ & $<0.05$ & 3.4 & (2.8 to 4.0$)$ & $<0.05$ \\
\hline & CS B vs CS BM & 1.5 & (0.8 to 2.2$)$ & $<0.05$ & 0.7 & (0.1 to 1.3$)$ & $<0.05$ \\
\hline \multicolumn{8}{|c|}{ Culex quinquefasciatus TPRI } \\
\hline \multirow[t]{6}{*}{ Mud } & CS B vs EC & 4.4 & (3.4 to 5.8 ) & $<0.05$ & $\dagger$ & + & t \\
\hline & CS BM vs EC & 5.6 & (4.8 to 6.3 ) & $<0.05$ & 7.5 & (6.4 to 8.9$)$ & $<0.05$ \\
\hline & Lambda vs EC & 1.2 & (0.6 to 1.7$)$ & $<0.05$ & 1.6 & (1.0 to 2.1$)$ & $<0.05$ \\
\hline & CS B vs Lambda & 3.2 & (2.2 to 4.6$)$ & $<0.05$ & $\dagger$ & 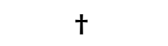 & + \\
\hline & CS BM vs Lambda & 4.4 & (3.8 to 5.2 ) & $<0.05$ & 6.0 & (4.9 to 7.4$)$ & $<0.05$ \\
\hline & CS B vs CS BM & -1.2 & $(-2.4$ to 0.4$)$ & $n / s$ & $\dagger$ & $\dagger$ & $t$ \\
\hline \multirow[t]{6}{*}{ Concrete } & CS B vs EC & 7.4 & (6.7 to 8.4$)$ & $<0.05$ & 8.4 & (7.5 to 9.4$)$ & $<0.05$ \\
\hline & CS BM vs EC & 6.0 & $(-0.2$ to 6.4$)$ & $n / s$ & 7.2 & (6.7 to 7.8$)$ & $<0.05$ \\
\hline & Lambda vs EC & 4.2 & (3.8 to 4.5 ) & $<0.05$ & 4.6 & (4.3 to 4.9 ) & $<0.05$ \\
\hline & CS B vs Lambda & 3.2 & (2.4 to 4.3 ) & $<0.05$ & 3.8 & (2.9 to 4.8$)$ & $<0.05$ \\
\hline & CS BM vs Lambda & 1.8 & $(-4.4$ to 2.4$)$ & $\mathrm{n} / \mathrm{s}$ & 2.7 & (2.1 to 3.3$)$ & $<0.05$ \\
\hline & CS B vs CS BM & 1.4 & $(0.5$ to 7.5$)$ & $<0.05$ & 1.2 & $(0.2$ to 2.2$)$ & $<0.05$ \\
\hline \multicolumn{8}{|c|}{ Culex quinquefasciatus Muheza } \\
\hline \multirow[t]{6}{*}{ Mud } & CS B vs EC & 3.2 & (2.7 to 3.9$)$ & $<0.05$ & 5.8 & (4.2 to 9.8$)$ & $<0.05$ \\
\hline & CS BM vs EC & 3.0 & (2.5 to 3.6$)$ & $<0.05$ & 5.1 & (4.4 to 6.2 ) & $<0.05$ \\
\hline & Lambda vs EC & $\dagger$ & $\dagger$ & $\dagger$ & -0.3 & $(-0.9$ to 0.3$)$ & $\mathrm{n} / \mathrm{s}$ \\
\hline & CS B vs Lambda & $t$ & $\dagger$ & $\dagger$ & 6.1 & (4.2 to 10.2 ) & $<0.05$ \\
\hline & CS BM vs Lambda & $\dagger$ & $\dagger$ & $\dagger$ & 5.5 & (4.6 to 6.6$)$ & $<0.05$ \\
\hline & CS B vs CS BM & 0.2 & $(-0.5$ to 0.9$)$ & $n / s$ & 0.7 & $(-1.2$ to 4.6$)$ & $n / s$ \\
\hline \multirow[t]{6}{*}{ Concrete } & CS B vs EC & 3.9 & (3.0 to 4.6$)$ & $<0.05$ & 5.2 & (4.2 to 6.0$)$ & $<0.05$ \\
\hline & CS BM vs EC & 3.3 & (2.9 to 3.7 ) & $<0.05$ & 4.3 & (3.7 to 4.8 ) & $<0.05$ \\
\hline & Lambda vs EC & 0.1 & $(-0.3$ to 0.5$)$ & $n / s$ & 0.4 & $(-0.1$ to 0.9$)$ & $\mathrm{n} / \mathrm{s}$ \\
\hline & CS B vs Lambda & 3.8 & (3.0 to 4.6 ) & $<0.05$ & 4.7 & (3.9 to 5.8 ) & $<0.05$ \\
\hline & CS BM vs Lambda & 3.2 & (2.8 to 3.7 ) & $<0.05$ & 3.9 & (3.3 to 4.4 ) & $<0.05$ \\
\hline & CS B vs CS BM & 0.6 & $(-0.2$ to 1.4$)$ & $n / s$ & 0.8 & $(0.0$ to 1.9$)$ & $n / s$ \\
\hline
\end{tabular}

tIndicates that statistical models produced estimates outside the study period for one or more of the treatments or their $95 \% \mathrm{Cl}$ and treatment differences cannot therefore be estimated.

ranging between $39-49 \%$ for CS formulations and 36$43 \%$ for EC (Table 7). Blood-feeding inhibition was similar for both CS and EC formulations over the trial. The mortality-feeding index (total blood-fed dead/total blood-fed) - (total unfed dead/total unfed) was 0.08 and 0.05 for CS 'B' and 0.08 and 0.03 for CS 'BM' on concrete and mud walled huts compared with 0.07 for EC and 0.15 for the unsprayed hut (mud walls). For all treatments the mortality-feeding index was close to 0 indicating mosquitoes had an equal chance of surviving whether fed or unfed.

Fumigant activity tested in small cages resulted in $100 \%$ mortality of $A n$. arabiensis F1 one week and two months after spraying for CS 'B', 'BM' and EC formulations. A large decrease to $42 \%$ fumigant mortality was recorded after three months for CS 'BM' (concrete) with fumigant mortality less than $10 \%$ for all other treatments. 

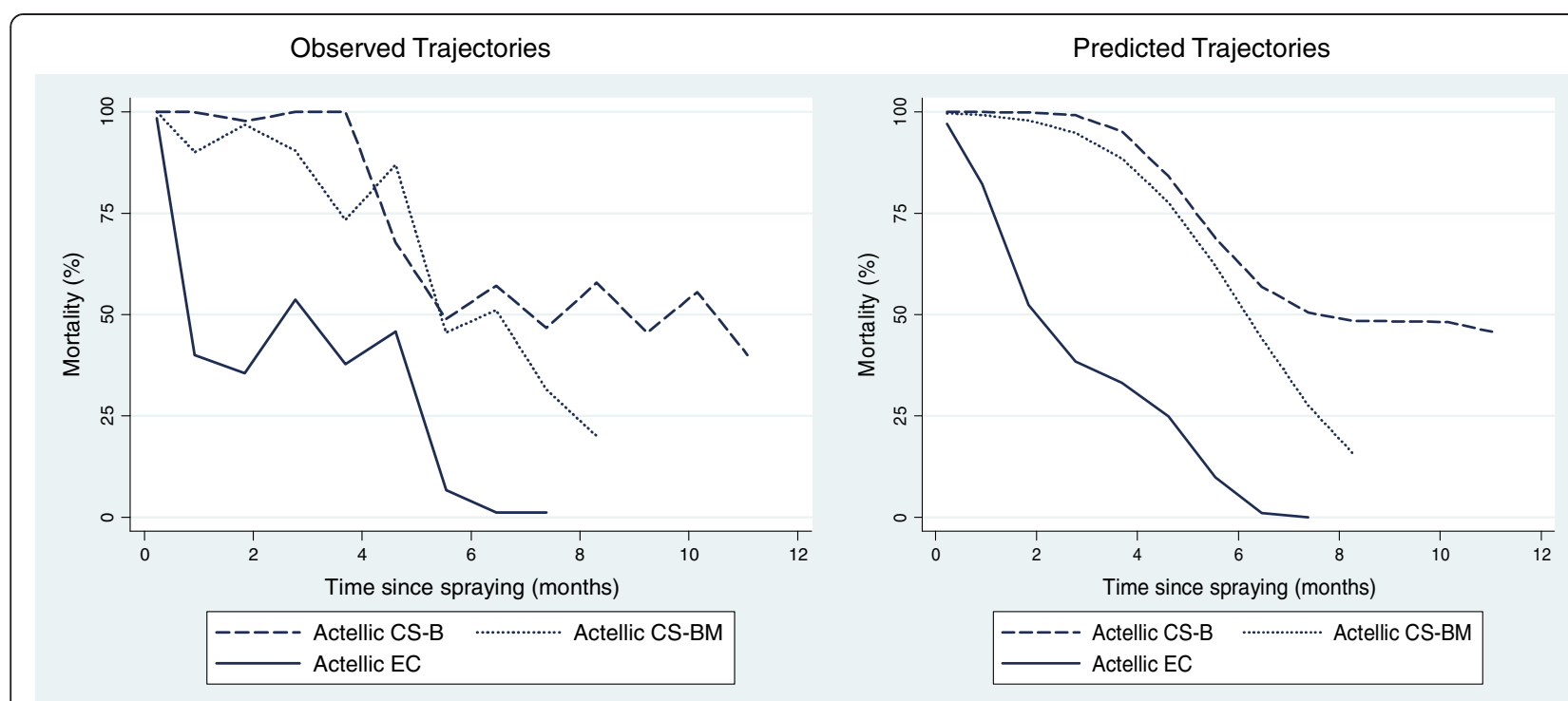

Figure 1 Mortality of Anopheles arabiensis dondotha on mud blocks after one-hour bioassays. Mud blocks were sprayed with p-methyl CS 'B', CS 'BM', and EC and tested at monthly intervals. Mortality for unsprayed blocks was $<15 \%$ for all bioassays.

\section{Supplementary explanatory bioassays in experimental} huts

The walls and ceiling of the p-methyl EC hut were covered with untreated plastic sheeting between months 12-13. This was done to investigate the possibility of mosquito movement between huts, picking up a lethal dosage of p-methyl CS before exiting, flying into the EC hut and dying. All other huts were left uncovered. Mortality for the covered EC hut was $29 \%$, which was greater than the unsprayed hut, $1 \%(\mathrm{P}=0.001)$ but less than huts sprayed with CS 'B', 65\%, 78\% and CS 'BM', 67\%, 74\% with concrete and mud walls respectively $(\mathrm{P}=0.001)$ (Table 8$)$. The

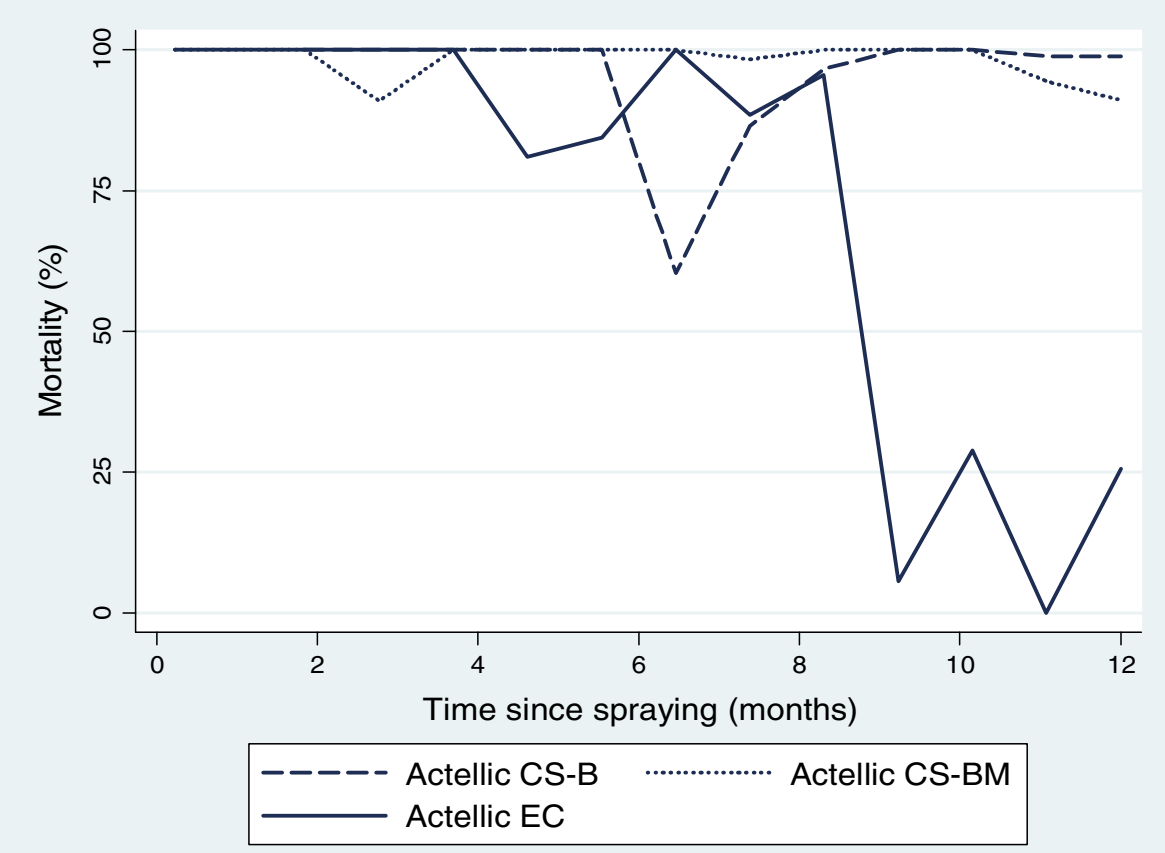

Figure 2 Mortality of Anopheles arabiensis dondotha on plywood blocks after one-hour bioassays. Plywood blocks were sprayed with p-methyl CS 'B', CS 'BM', and EC and tested at monthly intervals. Mortality for unsprayed blocks was $<15 \%$ for all bioassays. 


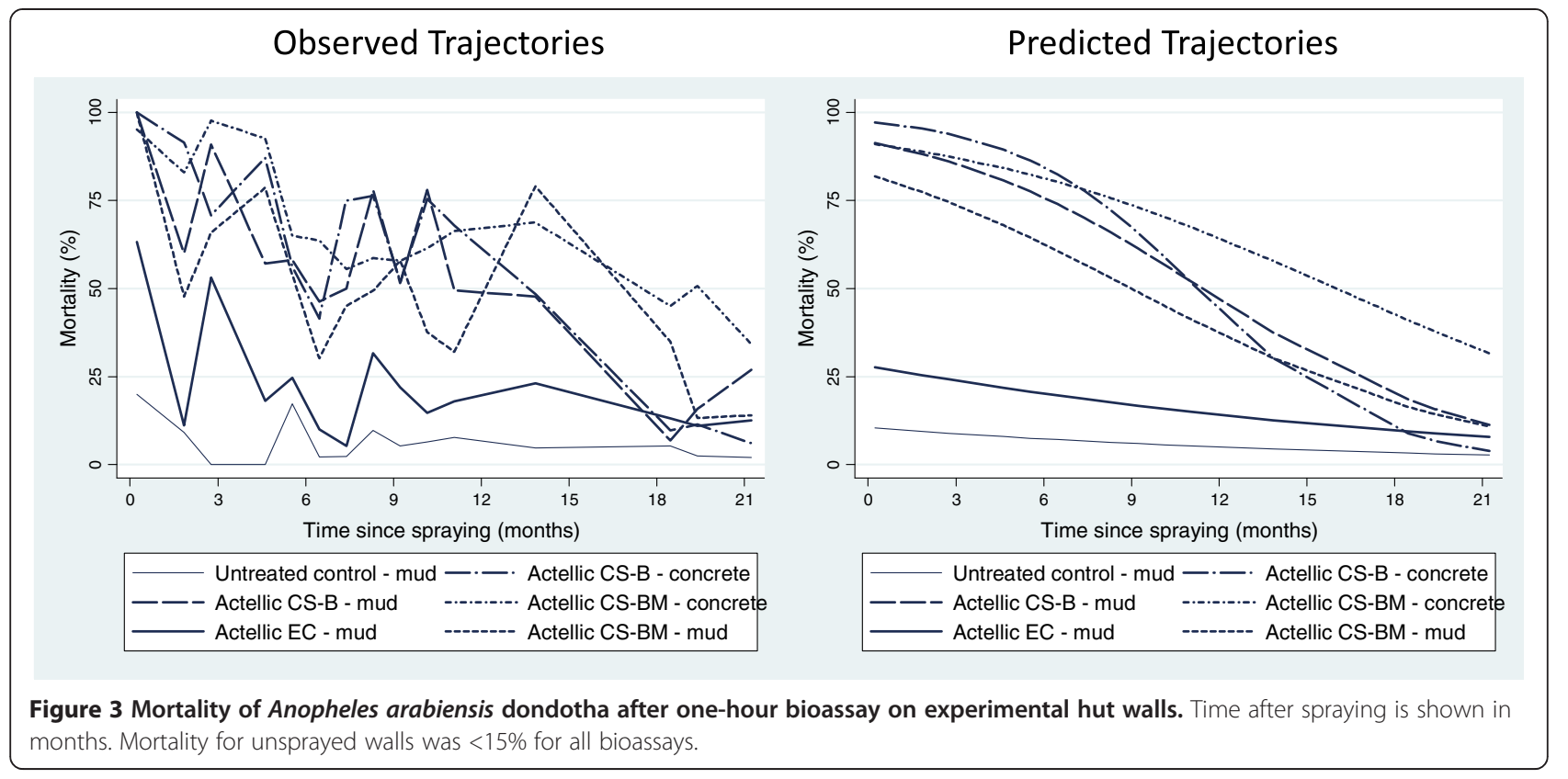

proportion of An. arabiensis that blood-fed was significantly higher in the covered EC hut (63\%), than for CS formulations $(19-38 \%, \mathrm{P}<0.05)$ but was less than the unsprayed hut $94 \%(\mathrm{P}=0.001)$.

To determine the relative contribution of the sprayed mud and concrete walls to mortality of An. arabiensis the palm thatch ceiling was covered with unsprayed plastic sheeting every second week between months 15-16. As the palm thatch ceiling remained highly insecticidal over the duration of the study (Figure 4) the hypothesis was that it masked any differences in efficacy between the concrete and mud walls (Figure 3). The covering of the ceiling had little impact on overall mortality trends for the EC hut (mud) with $43 \%$ mortality when uncovered and $46 \%$ covered ( $\mathrm{P}=0.255)$ (Table 8). For both CS 'B' and CS 'BM' any differences in mortality after covering the ceiling were small for both mud and concrete huts.

Extended cone bioassays of up to 12 hours were undertaken, as may occur when mosquitoes enter a house early in the evening to blood-feed and subsequently rest on treated surfaces until the following morning before exiting. With one-hour exposure, four months after spraying the CS 'B' and CS 'BM' killed a far greater proportion $(\mathrm{P}=0.001)$ of An. arabiensis than $\mathrm{EC}$, with mortality $18 \%$ for EC compared with $57 \%$ and $79 \%$ for CS 'B' and CS 'BM' (Figure 7). With longer exposure of two hours, the EC killed $88 \%$ of An. arabiensis compared with $100 \%$ for CS formulations. A similar trend

Table 5 Estimated time (months) for mortality to decrease to 80 and $\mathbf{5 0 \%}$ for Anopheles arabiensis dondotha (pyrethroid susceptible), tested on sprayed experimental hut walls (concrete and mud) and ceiling (thatch)

\begin{tabular}{|c|c|c|c|c|c|}
\hline \multirow[t]{2}{*}{ Insecticide } & \multirow[t]{2}{*}{ Substrate } & \multicolumn{2}{|c|}{ Estimated time to $80 \%$ mortality } & \multicolumn{2}{|c|}{ Estimated time to $\mathbf{5 0} \%$ mortality } \\
\hline & & Time (months) & $95 \% \mathrm{Cl}$ & Time (months) & $95 \% \mathrm{Cl}$ \\
\hline \multicolumn{6}{|l|}{ Hut walls } \\
\hline P-methyl EC & Mud & $\dagger$ & $\dagger$ & $\dagger$ & $\dagger$ \\
\hline \multirow[t]{2}{*}{ P-methyl CS B } & Concrete & 7.0 & (5.4 to 8.3$)$ & 11.3 & (10.2 to 12.4$)$ \\
\hline & Mud & 4.8 & (1.9 to 6.9$)$ & 11.4 & (9.9 to 13.0$)$ \\
\hline \multirow[t]{2}{*}{ P-methyl CS BM } & Concrete & 6.6 & (3.0 to 9.0$)$ & 16.0 & (13.5 to 20.6$)$ \\
\hline & Mud & 0.9 & († to 4.4$)$ & 9.0 & (6.4 to 11.0$)$ \\
\hline \multicolumn{6}{|l|}{ Hut ceilings } \\
\hline P-methyl EC & Thatch & $\dagger$ & $t$ & 2.4 & († to 6.1 ) \\
\hline P-methyl CS B & Thatch & 8.4 & (7.4 to 9.4$)$ & 12.0 & (11.2 to 12.7$)$ \\
\hline P-methyl CS BM & Thatch & 10.8 & (9.9 to 11.7 ) & 14.4 & (13.7 to 15.2$)$ \\
\hline
\end{tabular}

†Indicates that statistical models produced estimates outside the study period: in all cases estimates were lower than the specified mortality $(50$ or $80 \%$, respectively) throughout the entire study period. 


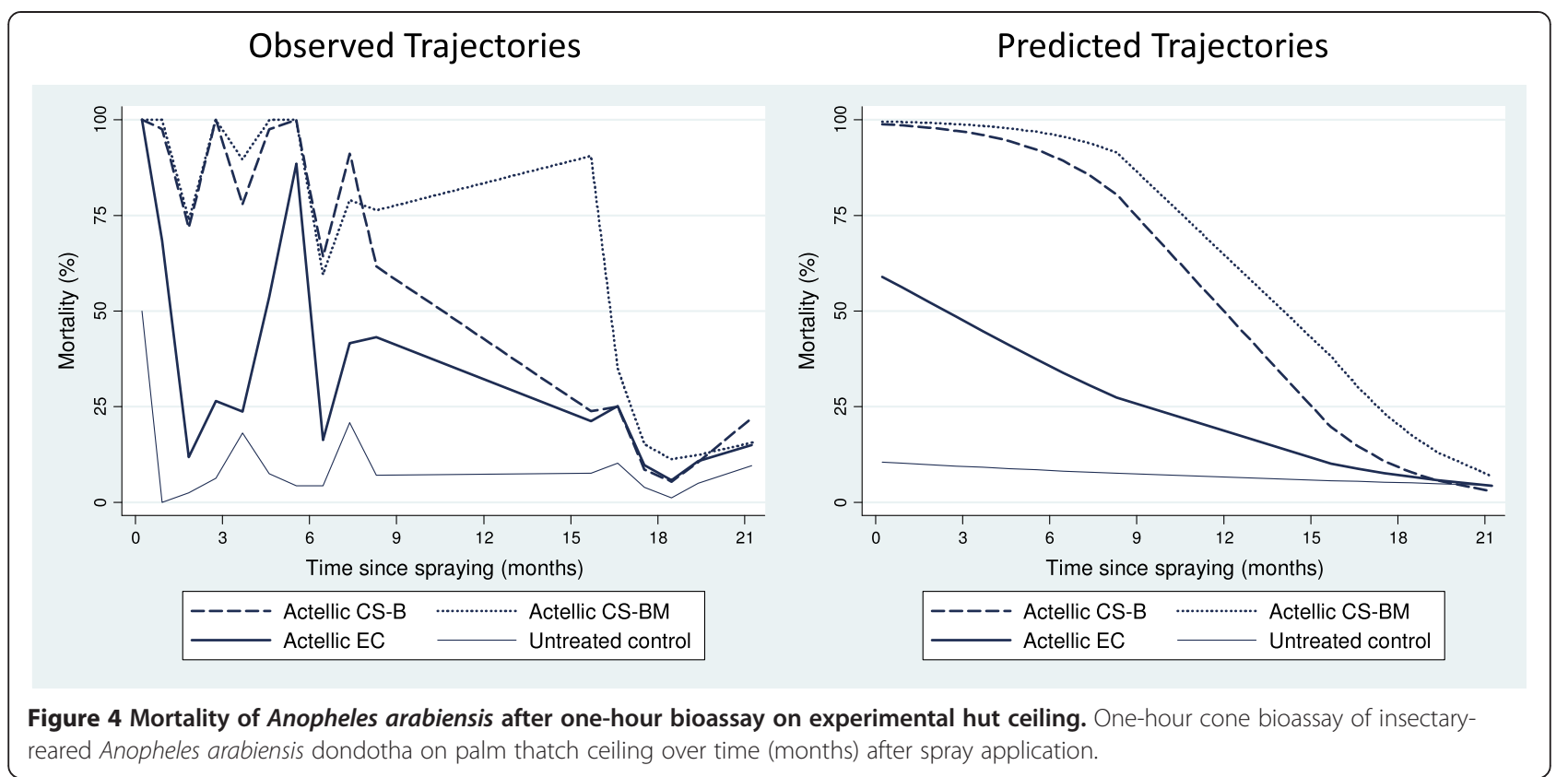

was observed after ten months as the EC killed 15\% with one-hour exposure but killed 73\% with a four-hour exposure compared with $80 \%$ for CS 'BM' $(\mathrm{P}=0.401)$ and 97\% for CS 'B' $(\mathrm{P}=0.014)$. After 17 months mortality was low for both CS 'B' (20\%) and EC (20\%) with onehour exposure but increased to $52 \%$ for EC, $72 \%$ CS 'B', and $98 \%$ for CS 'BM' with 12-hour exposure.

\section{Discussion}

Laboratory bioassays showed that p-methyl CS 'B' and CS 'BM' formulations were effective at killing high proportions
(>80\%) of An. arabiensis and Cx. quinquefasciatus for significantly longer than the EC formulation on mud, concrete and plywood substrates. The most important improvement was observed on mud. The EC was ineffective on mud and killed $>80 \%$ of An. arabiensis and Cx. quinquefasciatus for one month or less. In contrast, the best performing CS formulation killed $>80 \%$ of An. arabiensis for five months and sustained control above $50 \%$ for longer than seven months. Similar longevity was observed in The Gambia where p-methyl CS sprayed in village houses persisted for at least five months (when testing was ended) on mud and

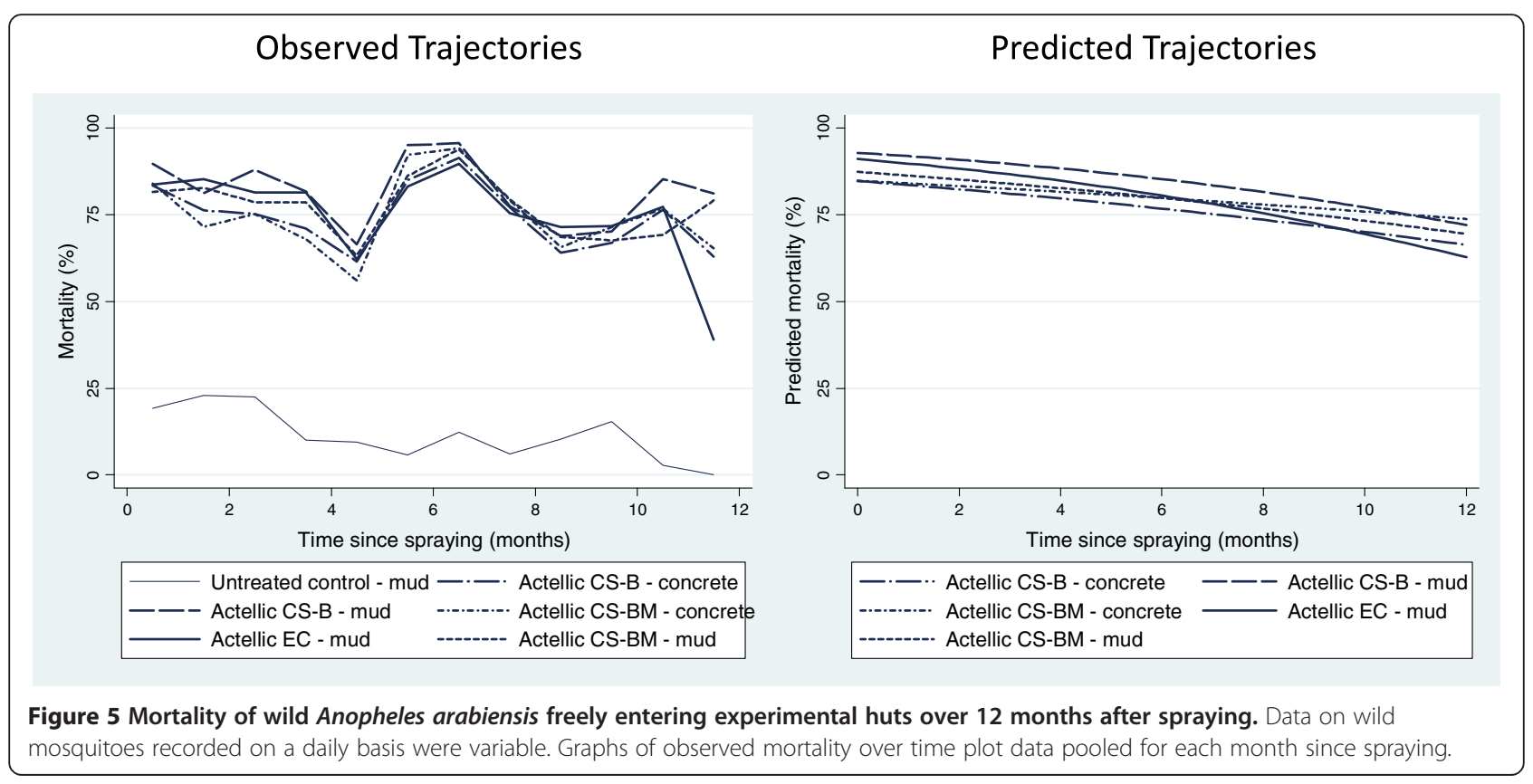


Table 6 Estimated mortality (\%) three, six, nine and twelve months after spraying for wild mosquitoes collected in insecticide treated huts

\begin{tabular}{|c|c|c|c|c|c|}
\hline \multirow[b]{2}{*}{ Insecticide } & \multirow[b]{2}{*}{ Substrate } & \multicolumn{4}{|c|}{$\%$ Mortality $(95 \% \mathrm{Cl})$} \\
\hline & & 3 months & 6 months & 9 months & 12 months \\
\hline \multirow[t]{2}{*}{ P-methyl EC } & Mud & 86.6 & 80.5 & 72.5 & 62.8 \\
\hline & & (83.9 to 89.4) & (77.8 to 83.3 ) & (67.9 to 77.2 ) & (54.4 to 71.2$)$ \\
\hline \multirow[t]{4}{*}{ P-methyl CS B } & Concrete & 81.0 & 76.8 & 71.8 & 66.3 \\
\hline & & (77.7 to 84.4 ) & (73.7 to 79.8 ) & (67.1 to 76.6 ) & (58.3 to 74.3 ) \\
\hline & Mud & 89.6 & 85.3 & 79.4 & 72.0 \\
\hline & & (87.3 to 92.0$)$ & (82.9 to 87.6 ) & (75.4 to 83.4 ) & (64.5 to 79.6$)$ \\
\hline \multirow[t]{4}{*}{ P-methyl CS BM } & Concrete & 82.5 & 79.8 & 76.9 & 73.8 \\
\hline & & (79.3 to 85.6 ) & (77.1 to 82.6 ) & (72.9 to 81.0 ) & (67.0 to 80.5$)$ \\
\hline & Mud & 83.9 & 79.8 & 75.0 & 69.5 \\
\hline & & (80.9 to 86.9) & (77.1 to 82.6 ) & (70.6 to 79.4 ) & (62.0 to 77.0 ) \\
\hline
\end{tabular}

Estimates are adjusted for sleeper and account for similarities among mosquitoes entering huts on the same day and potential behavioural clustering.

painted walls [28]. Mud is a problematic substrate for IRS owing to loss of available insecticide due to sorption. Early work in Tanzania in the 1960s characterized the performance of organophosphates and carbamates on various types of soil and showed rapid loss of efficacy on several types of mud, while on less porous substrates, such as wood, high levels of mortality were recorded over several months $[29,30]$. In the present study, microencapsulation substantially improved the surface bioavailability of p-methyl on mud. Mud or adobe is still a common wall material in rural, low-income areas of
Africa. In Tanzania in 2010, 78\% of houses were constructed from a form of mud; the most common types being mud plaster (27\%), sun-dried mud bricks (28\%) and burnt mud bricks (23\%) [25].

Both CS formulations showed improved longevity over EC on concrete and wood substrates in bioassays. The alkaline $\mathrm{Ph}$ of concrete can rapidly degrade insecticides commonly used for IRS, particularly pyrethroids, resulting in reduced residual efficacy [17]. In laboratory bioassays on plywood, CS formulations lasted for several months longer than the EC, and killed $>80 \%$ of An. arabiensis 12

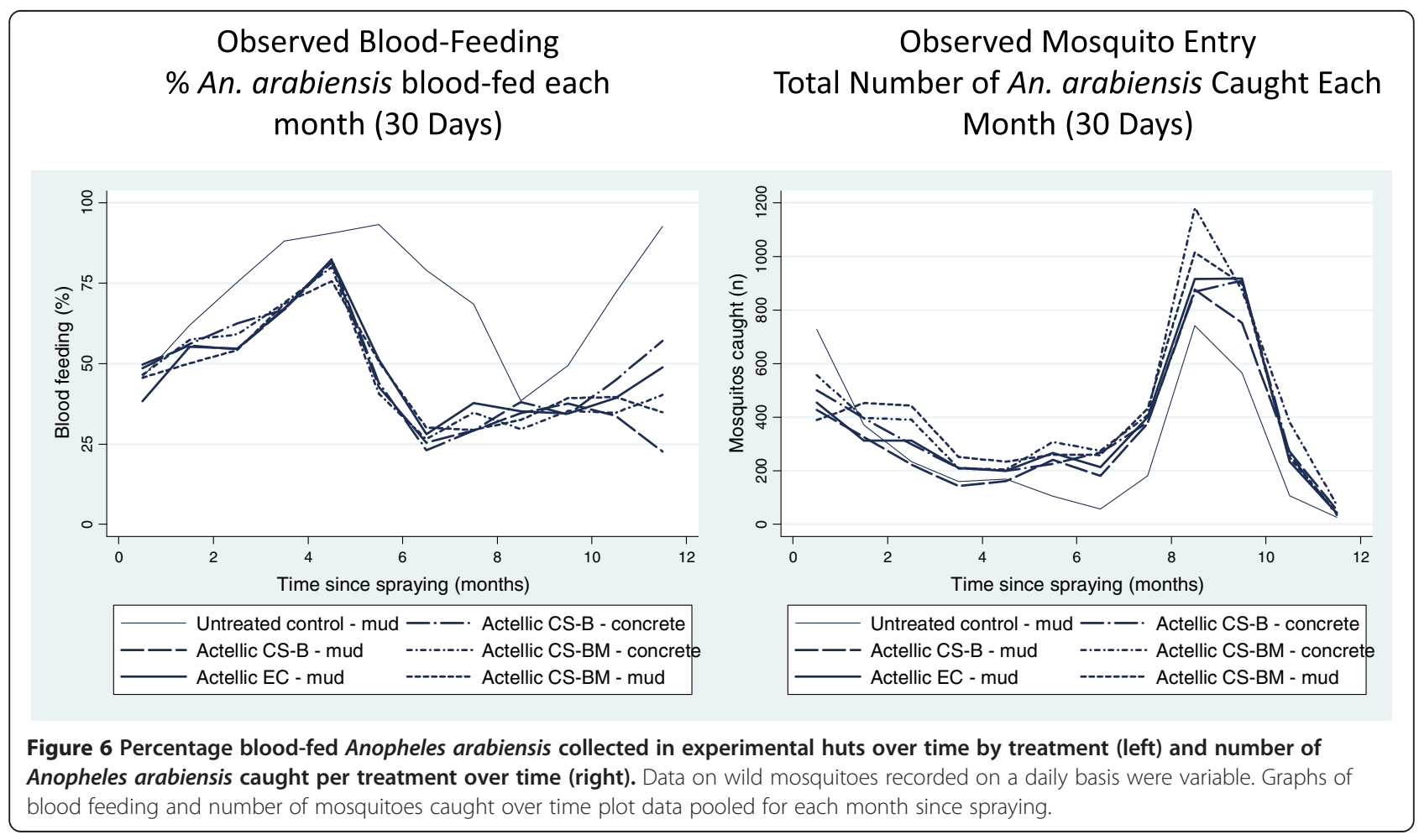


Table 7 Estimated blood feeding (\%) three, six, nine and twelve months after spraying for wild mosquitoes collected in insecticide treated huts

\begin{tabular}{|c|c|c|c|c|c|c|c|c|c|}
\hline \multirow[t]{3}{*}{ Insecticide } & \multirow[t]{3}{*}{ Substrate } & \multicolumn{2}{|c|}{3 months } & \multicolumn{2}{|c|}{6 months } & \multicolumn{2}{|c|}{9 months } & \multicolumn{2}{|c|}{12 months } \\
\hline & & Blood fed (\%) & BFI (\%) & Blood fed (\%) & $\overline{\text { BFI (\%) }}$ & Blood fed (\%) & BFI (\%) & Blood fed (\%) & $\overline{\text { BFI (\%) }}$ \\
\hline & & $(95 \% \mathrm{Cl})$ & & $(95 \% \mathrm{Cl})$ & & $(95 \% \mathrm{Cl})$ & & $(95 \% \mathrm{Cl})$ & \\
\hline \multirow[t]{2}{*}{ Untreated control } & Mud & 90 & - & 81 & - & 57 & - & 93 & - \\
\hline & & (87 to 93 ) & & (77 to 85 ) & & (52 to 63$)$ & & (86 to 100 ) & \\
\hline \multirow[t]{2}{*}{ P-methyl EC } & Mud & 69 & 24 & 52 & 36 & 33 & 43 & 67 & 29 \\
\hline & & (64 to 74 ) & & (47 to 57$)$ & & (28 to 37 ) & & (49 to 84 ) & \\
\hline \multirow[t]{4}{*}{ P-methyl CS B } & Concrete & 71 & 22 & 49 & 40 & 32 & 44 & 84 & 9 \\
\hline & & (66 to 76$)$ & & (44 to 54 ) & & (28 to 37 ) & & (73 to 96$)$ & \\
\hline & Mud & 66 & 26 & 50 & 39 & 31 & 46 & 47 & 49 \\
\hline & & (61 to 72 ) & & (44 to 55$)$ & & (26 to 35$)$ & & (26 to 69$)$ & \\
\hline \multirow[t]{4}{*}{ P-methyl CS BM } & Concrete & 68 & 24 & 48 & 41 & 29 & 49 & 63 & 33 \\
\hline & & (63 to 73 ) & & (43 to 53 ) & & (25 to 33 ) & & (45 to 81 ) & \\
\hline & Mud & 67 & 26 & 49 & 39 & 31 & 46 & 63 & 32 \\
\hline & & (61 to 72 ) & & (44 to 54 ) & & (27 to 35 ) & & (44 to 82 ) & \\
\hline
\end{tabular}

Estimates are adjusted for sleeper and account for similarities among mosquitoes entering huts on the same day and potential behavioural clustering. $\mathrm{BFI}=$ blood-feeding inhibition compared to the untreated control.

months after spraying. Wood is relatively non-porous with a tendency for long residual bioavailability of organophosphates and pyrethroids [29,31].

Cone bioassays on mud and concrete experimental hut walls showed similar findings to laboratory results and showed that both CS formulations were effective for significantly longer than the EC. For all bioassays in the laboratory and experimental huts an exposure time of 60 minutes was used rather than the standard WHOPES 30 minutes exposure. It is likely that the residual duration of action would be shorter if tested using WHOPES guidelines.

Results for free-flying, wild An. arabiensis showed that huts sprayed with p-methyl CS formulations maintained

Table 8 Supplementary experimental hut results for percentage mortality and blood-feeding, 13-16 months after spraying

\begin{tabular}{|c|c|c|c|c|c|c|c|}
\hline Time after spraying & $\begin{array}{l}\text { Outcome } \\
\text { measures }\end{array}$ & $\begin{array}{l}\text { Untreated } \\
\text { (Mud) }\end{array}$ & $\begin{array}{c}\text { CS-B } \\
\text { (Concrete) }\end{array}$ & $\begin{array}{c}\text { CS-BM } \\
\text { (Concrete) }\end{array}$ & $\begin{array}{l}\text { CS-B } \\
\text { (Mud) }\end{array}$ & $\begin{array}{l}\text { CS-BM } \\
\text { (Mud) }\end{array}$ & $\begin{array}{c}\text { EC } \\
\text { (Mud) }\end{array}$ \\
\hline \multirow{5}{*}{$\begin{array}{l}13 \text { Months (EC Walls \& Ceiling } \\
\text { Covered) }\end{array}$} & Total Caught & 92 & 181 & 204 & 143 & 170 & 115 \\
\hline & $\%$ Mortality & 1 & 65 & 67 & 78 & 74 & 29 \\
\hline & $95 \% \mathrm{Cl}$ & (1 to 6$)$ & (51 to 77$)$ & $(45-83)$ & $(63-88)$ & $(61-83)$ & $(13-51)$ \\
\hline & $\%$ Blood-fed & 94 & 32 & 30 & 19 & 38 & 63 \\
\hline & $\% \mathrm{BFI}$ & - & 66 & 68 & 80 & 60 & 33 \\
\hline \multirow[t]{5}{*}{ 15-16 Months (Ceiling Uncovered) } & Total Caught & 411 & 592 & 870 & 576 & 685 & 629 \\
\hline & $\%$ Mortality & 5 & 34 & 42 & 48 & 63 & 43 \\
\hline & $95 \% \mathrm{Cl}$ & $(2-12)$ & $(27-42)$ & $(33-51)$ & $(36-59)$ & $(46-77)$ & $(31-55)$ \\
\hline & $\%$ Blood-fed & 59 & 48 & 53 & 51 & 42 & 52 \\
\hline & $\% \mathrm{BFI}$ & - & 19 & 10 & 14 & 29 & 12 \\
\hline \multirow[t]{5}{*}{ 15-16 Months (Ceiling Covered) } & Total Caught & 303 & 557 & 455 & 390 & 498 & 580 \\
\hline & $\%$ Mortality & 7 & 48 & 49 & 49 & 53 & 46 \\
\hline & $95 \% \mathrm{Cl}$ & $(3-15)$ & $(41-55)$ & $(38-60)$ & $(38-59)$ & $(41-64)$ & $(37-55)$ \\
\hline & $\%$ Blood-fed & 69 & 47 & 46 & 51 & 45 & 54 \\
\hline & $\% \mathrm{BFI}$ & - & 32 & 33 & 26 & 35 & 22 \\
\hline
\end{tabular}

During month 13 the walls and ceiling of the hut sprayed with p-methyl EC were covered with plastic sheeting. Between months 15 and 16 the treated walls of every hut were covered with plastic sheeting for one out of every two weeks. Data are grouped according to whether the walls were covered or uncovered. $\mathrm{BFI}=$ blood-feeding inhibition compared to untreated control. 


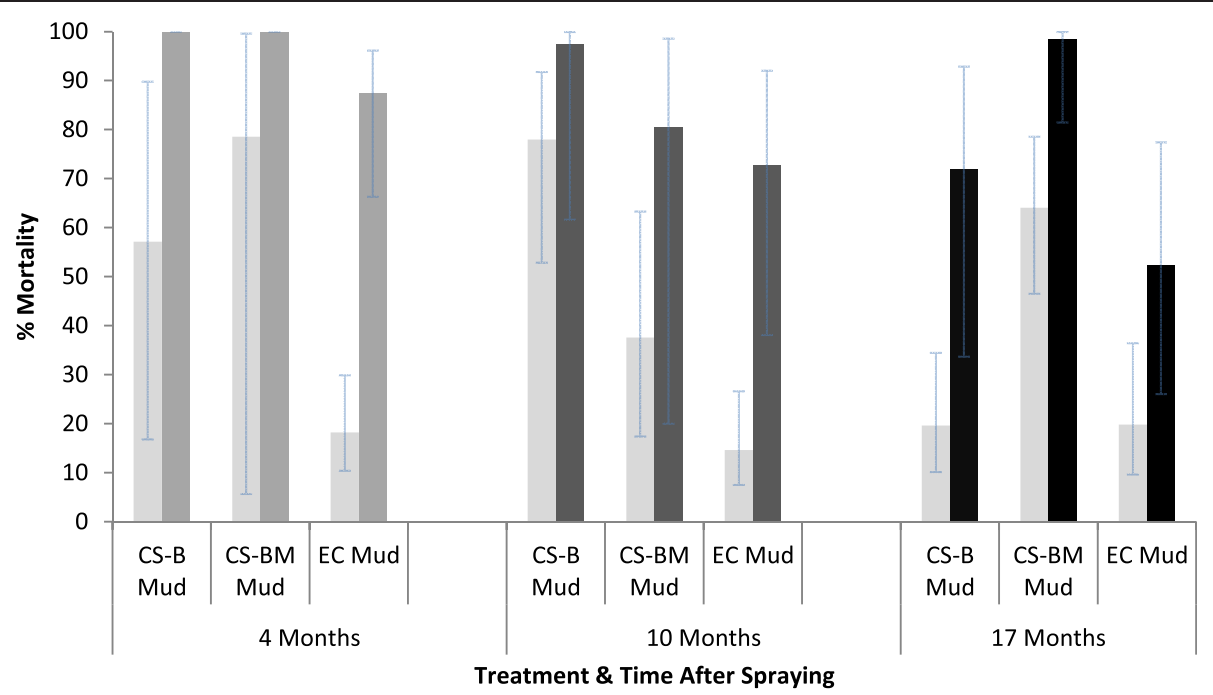

Figure 7 Results of extended duration bioassays on walls of experimental huts. Percentage mortality of insectary-reared Anopheles arabiensis dondotha following cone bioassay with standard exposure time of one hour (light bars) and extended exposure (darker bars) of two hours (four months), four hours (ten months), 12 hours (17 months) on sprayed mud walls. Mortality for unsprayed walls was $<20 \%$ for all bioassays.

high rates of mortality for up to 12 months after spraying. This finding is comparable to that in Benin where $1 \mathrm{~g} / \mathrm{sq} \mathrm{m}$ of p-methyl sprayed in mud and concrete experimental huts killed around $75 \%$ of wild free-flying An. gambiae s.s. ten months after spraying [32].

In Tanzania, there was an increase in mortality for all formulations five to seven months after spraying between May-July. This was the cool season when mean night-time temperature outdoors dropped to $20^{\circ} \mathrm{C} \mathrm{com}$ pared with $24^{\circ} \mathrm{C}$ inside the experimental huts (USB Wireless Touchscreen Weather Forecaster, Maplin, UK). This may have resulted in longer indoor resting times, which would explain the increase in mortality during this three-month period. It has been reported elsewhere that at higher altitude where differences between indoor and outdoor temperature are greatest, indoor resting is more common [33,34].

An unexpected finding was that the EC formulation matched the performance of the CS against wild free-flying An. arabiensis despite being considered by WHOPES to have an effective duration of only two to three months $[17,32]$. Recent studies in Ghana on painted cement, and Mozambique on several surfaces, showed high levels of mortality for the EC formulation > four months after spraying, indicating that the EC can remain effective for a relatively long duration [35]. In this study the EC maintained high levels of mortality for wild free-flying An. arabiensis but paradoxically showed poor performance in one-hour cone bioassay on hut walls only weeks after spraying. Several explanations were postulated:

Mosquito resting location: Mortality in the EC hut may have been generated by tarsal contact with palm thatch ceiling, with mud walls providing a small proportion of overall mortality. Covering the ceiling with untreated plastic did not result in a decrease in mortality, indicating that mosquitoes were able to pick up a lethal dosage from treated mud walls.

Mosquito movement between huts: It was plausible that mosquitoes were picking up a lethal dosage of p-methyl CS before exiting through open verandahs, flying into the EC hut and falsely being recorded as killed by the EC. Covering all sprayed surfaces (walls and ceiling) with untreated plastic for one month (13 months after spraying) in the EC hut should have resulted in low mortality rates similar to an unsprayed hut if there was no movement of mosquitoes between huts. When covered, mortality was $29 \%$, which although slightly higher than the unsprayed hut, suggested that few mosquitoes were flying between huts. Throughout the trial mortality in the unsprayed control was $<20 \%$. This suggests that mortality was generated by insecticidal activity within each individual hut and any movement of mosquitoes between huts had a limited effect on mortality trends.

Mosquito resting duration: The standard exposure time as specified by WHO for IRS cone bioassay is 30 minutes, regardless of the insecticide [9]. This exposure time is probably suitable for excito-repellent insecticides such as pyrethroids and DDT. Resting times of blood-fed $A n$. gambiae on a wall sprayed with a non-irritant insecticide, such as p-methyl, may be longer than 30 minutes. For this study an exposure of one hour was selected for monthly bioassays with supplementary bioassays of up to 12 hours. In the EC hut the finding that one-hour bioassays killed a small proportion of An. arabiensis, while hut collections showed high levels of mortality may indicate that mosquitoes either, i) rested for a short time and exited before 
picking up lethal dosage or ii) rested for several hours. Extended cone bioassay of two hours after four months and four hours after ten months showed high levels of mortality for both EC and CS formulations. Anopheles arabiensis may have rested on treated surfaces for several hours overnight and may partially explain why EC mortality was similar to that of the CS formulations for wild, free-flying An. arabiensis. While this offers some understanding to why the EC was effective for a longer duration than expected, it does not provide a full explanation for this. As new insecticides are developed for IRS with low excitorepellency, WHOPES may have to revisit the standard 30 minutes exposure for IRS, if this period of exposure does not provide an accurate prediction of field performance.

The mortality-feeding index showed that unfed mosquitoes were equally likely to be killed by p-methyl as those blood-fed. The concept of IRS is to kill mosquitoes that blood-feed and then rest on treated surfaces while processing the blood meal. This finding indicates that some $A n$. arabiensis rested on hut surfaces before attempting to blood-feed and explains why there was some protective effect of p-methyl IRS [36]. There were apparent seasonal changes in percentage blood-feeding in the unsprayed hut. The periods of lowest proportion blood-fed coincided with peak mosquito densities during rice transplantation. It is likely that a larger proportion of newly emerged $A n$. arabiensis entered experimental huts from adjacent paddies for resting or sugar feeding, rather than host-seeking [37].

There was a fumigant effect of all formulations that killed a high proportion of mosquitoes in cage bioassays during the first two months after spraying. The microcapsules in the CS would have limited any fumigant effect because the majority of active ingredient is enclosed within the capsule membrane; however some active ingredient is also present in external solution. Slow release of active ingredient from microcapsules was sufficient for contact mortality but insufficient for a fumigant effect. Questionnaires of volunteers sleeping during the hut trial resulted in Actellic EC ranked consistently last in terms of odour appeal, with typical comments including, "Smells like cabbage and white spirit" or, "Not pleasant and produces irritation". The CS formulations ranked better, and were generally considered to be much milder than the EC, with comments such as, "Smells like cow insecticide, appealing as not too strong".

Of 17 African countries sprayed with PMI-funded IRS in 2012, only one was classified as having pyrethroid susceptible anophelines; the remainder had confirmed or emerging resistance [10]. The Global Plan for Insecticide Resistance Management (GPIRM) states that in areas of pyrethroid resistance IRS rotations should be used with non-pyrethroid insecticides [38]. Despite added impetus from the IVCC there have been no new insecticides for
IRS and LLIN since the pyrethroids in the 1980s [11]. As a result, the majority of African PMI-funded IRS programmes are currently spraying IRS with bendiocarb which has a short residual efficacy of only two to six months and is relatively expensive [10,17]. In Malawi, where resistance to both pyrethroids and carbamates was detected, p-methyl EC was sprayed in 2011, but "although effective, the high unit cost substantially increased the IRS costs and PMI subsequently suspended direct support due to increased costs" [39]. Long-lasting p-methyl CS formulations should be more cost-effective than both p-methyl $\mathrm{EC}$ and bendiocarb, but this estimation is sensitive to both the duration of efficacy and the relative cost per unit area sprayed. Use of p-methyl IRS + pyrethroid LLIN is preferential for resistance management to pyrethroid IRS + pyrethroid LLINs as p-methyl and pyrethroids have different modes of action which should result in redundant killing of mosquitoes resistant to a single insecticide [40]. Crossresistance of organophosphates and carbamates due to altered acetylcholinesterase (AChE) target site is present at low frequency in limited parts of west and central Africa and may increase in frequency as a result of current IRS programmes using bendiocarb. Nevertheless, IRS with p-methyl CS should prove an effective solution for control of pyrethroid resistant An. gambiae and, having received recent recommendation from WHO [41], is a welcome addition to the limited portfolio of long-lasting IRS.

\section{Ethical approval}

Ethical approval was granted from the review boards of LSHTM (5256) and Tanzania National Institute of Medical Research (NIMR/HQ/R.8c/Vol.I/24).

\section{Competing interests}

The authors declare that they have no competing interests.

\section{Authors' contributions}

RMO participated in the study design, data collection and analysis and drafted the manuscript. JK participated in the study design, data collection and helped to draft parts of the manuscript. RJ performed the statistical analysis and provided critical comments on the manuscript. EF and JM participated in data collection and was involved in drafting parts of the manuscript. FWM participated in study design and critically revised the manuscript. MWR participated in study design, analysis and multiple revisions of the manuscript. All authors read and approved the final manuscript.

\section{Acknowledgements}

$\mathrm{RMO}$ and MR are members of the Malaria Centre of the London School of Hygiene and Tropical Medicine http:/malaria.Ishtm.ac.uk/. The authors thank Andy Bywater and Mark Birchmore of Syngenta for supplying samples and for technical support; Dave Malone and Robert Sloss for technical

programme support (IVCC, Liverpool, UK). We are grateful to C Masenga, A Mtui, E Philipo, H Temba, P Anthony, A Sanga, R Athuman, K Ezekia for field and laboratory assistance.

\section{Author details}

${ }^{1}$ Department of Disease Control, London School of Hygiene and Tropical Medicine (LSHTM), London, UK. '² Department of Entomology and Parasitology, Kilimanjaro Christian Medical University College (KCMUCo) of Tumaini University, Moshi, Kilimanjaro, Tanzania. ${ }^{3}$ Department of Entomology, 
Pan-African Malaria Vector Research Consortium, (PAMVERC), Moshi, Kilimanjaro, Tanzania.

Received: 28 November 2013 Accepted: 12 January 2014 Published: 29 January 2014

\section{References}

1. Pluess B, Tanser FC, Lengeler C, Sharp BL: Indoor residual spraying for preventing malaria. Cochrane Database Syst Rev 2010:CD006657.

2. Griffith M: The World-Wide Malaria Eradication Program, Presented in the Plenary Symposium on International Programs at the meeting of the Entomological Society of America in New Orleans, November 30, 1965. 1965.

3. Molineaux L, Gramiccia G: The Garki Project : research on the epidemiology and control of malaria in the Sudan Savanna of West Africa. Geneva: World Health Organization; 1980.

4. WHO: Implementation of indoor residual spraying of insecticides for malaria control in the WHO African Region Report. Geneva, Switzerland: World Health Organization; 2007.

5. Akhtar RL, Learmonth A: The resurgence of malaria in India 1965-76. GeoJournal 1977, 1(5):69-80.

6. Pinikahana J, Dixon RA: Trends in malaria morbidity and mortality in Sri Lanka. Indian J Malariol 1993, 30:51-55.

7. Mabaso ML, Sharp B, Lengeler C: Historical review of malarial control in southern African with emphasis on the use of indoor residual housespraying. Trop Med Int Health 2004, 9:846-856.

8. WHO: Indoor Residual Spraying; Use of indoor residual spraying for scaling up global malaria control and elimination. Geneva, Switzerland: WHO/HTM/ MAL/2006.1112; 2006.

9. WHO: Guidelines for testing mosquito adulticides for indoor residual spraying and treatment of mosquito nets. Geneva, Switzerland: WHO/CDS/NTD/ WHOPES/GCDPP/2006.2003; 2006

10. PMI: PMI Actual and Estimated Use of Insecticides for the Indoor Residual Spraying Program. 2012 [http://www.pmi.gov/technical/irs/ PMI_IRS_Insecticide_Trends_080112.xIsx]

11. Hemingway J, Beaty BJ, Rowland M, Scott TW, Sharp BL: The innovative vector control consortium: improved control of mosquito-borne diseases. Trends Parasitol 2006, 22:308-312.

12. WHO: WHO recommended insecticide products treatment of mosquito nets for malaria vector control. Geneva: World Health Organization; 2007.

13. WHOPES: Report of the 4th WHOPES Working Group Meeting WHO/HQ. Geneva: WHO/CDS/WHOPES/2001.2002; 2000.

14. Ranson H, N'Guessan R, Lines J, Moiroux N, Nkuni Z, Corbel V: Pyrethroid resistance in African anopheline mosquitoes: what are the implications for malaria control? Trends Parasitol 2011, 27:91-98.

15. Maharaj R, Mthembu DJ, Sharp BL: Impact of DDT re-introduction on malaria transmission in KwaZulu-Natal. S Afr Med J 2005, 95:871-874.

16. Sharp BL, Ridl FC, Govender D, Kuklinski J, Kleinschmidt I: Malaria vector control by indoor residual insecticide spraying on the tropical island of Bioko Equatorial Guinea. Malar J 2007, 6:52.

17. WHO: WHO recommended insecticides for indoor residual spraying against malaria vectors. 2013. http://www.who.int/whopes/Insecticides_IRS_Malaria_ 25_Oct_2013.pdf?ua=1.

18. U.N.E.P: Ridding the World of POPs: A Guide to the Stockholm Convention on Persistent Organic Pollutants. 2010.

19. Zaim M, Guillet P: Alternative insecticides: an urgent need. Trends Parasitol 2002, 18:161-163.

20. Mabbett T: Pirimiphos methyl - long term solution to the problem of stored product pests. Int Pest Contr 2002:203-204.

21. Nasir SM, Ahmad N, Shah MA, Azam CM: A large-scale evaluation of pirimiphos-methyl 25\% WP during 1980-1981 for malaria control in Pakistan. J Trop Med Hyg 1982, 85:239-244.

22. President's Malaria Initiative: Malawi and Zambia Malaria Operational Plan FY 2013. 2013.

23. PMI: President's Malaria Initiative Tanzania Malaria Operational Plan FY 2013. 2013.

24. Curtis CF, Myamba J, Wilkes TJ: Comparison of different insecticides and fabrics for anti-mosquito bednets and curtains. Med Vet Entomol 1996, 10:1-11.
25. TDHS: Tanzania Demographic and Health Survey 2010. National Bureau of Statistics, Dar es Salaam, Tanzania. Calverton, Maryland, USA: ICF Macro; 2011.

26. WHO: Manual for Indoor Residual Spraying Application of Residual Sprays for Vector Control. Geneva, Switzerland: WHO/CDS/NTD/WHOPES/GCDPP/ 2007.2003; 2007c.

27. Kitau J, Oxborough RM, Tungu PK, Matowo J, Malima RC, Magesa SM, Bruce J, Mosha FW, Rowland MW: Species shifts in the Anopheles gambiae complex: do LLINs successfully control Anopheles arabiensis? PLoS One 2012, 7:e31481.

28. Tangena JA, Adiamoh M, D'Alessandro U, Jarju L, Jawara M, Jeffries D, Malik N, Nwakanma D, Kaur H, Takken W, Lindsay SW, Pinder M: Alternative treatments for indoor residual spraying for malaria control in a village with pyrethroid- and DDT-resistant vectors in The Gambia. PLoS One 2013, 8:e74351.

29. Hadaway $A B$, Barlow $F$ : The toxicity of some organophosphorus compounds to adult Anopheles stephensi. Bull World Health Organ 1963, 28:55-61.

30. Hadaway $A B$, Barlow $F$ : The residual action of two organophosphorus compounds and a carbamate on dried muds. Bull World Health Organ 1963, 28:69-76.

31. Tseng LF, Chang WC, Ferreira MC, Wu CH, Rampao HS, Lien JC: Rapid control of malaria by means of indoor residual spraying of alphacypermethrin in the Democratic Republic of Sao Tome and Principe. Am J Trop Med Hyg 2008, 78:248-250.

32. Rowland M, Boko P, Odjo A, Asidi A, Akogbeto M, N'Guessan R: A new long-lasting indoor residual formulation of the organophosphate insecticide pirimiphos methyl for prolonged control of pyrethroidresistant mosquitoes: an experimental hut trial in Benin. PLoS One 2013, 8:e69516.

33. Paaijmans KP, Thomas MB: The influence of mosquito resting behaviour and associated microclimate for malaria risk. Malar J 2011, 10:183.

34. Manguin S: Biodiversity of malaria in the world, English completely updated. edn. Montrouge ; Esther [Esher], Surrey: John Libbey Eurotext; 2008.

35. Fuseini G, Ebsworth P, Jones S, Knight D: The efficacy of ACTELLIC 50 EC, pirimiphos methyl, for indoor residual spraying in Ahafo, Ghana: area of high vector resistance to pyrethroids and organochlorines. J Med Entomol 2011, 48:437-440.

36. Oxborough RM, Kitau J, Matowo J, Mndeme R, Feston E, Boko P, Odjo A, Metonnou CG, Irish S, N'Guessan R, Mosha FW, Rowland MW: Evaluation of indoor residual spraying with the pyrrole insecticide chlorfenapyr against pyrethroid-susceptible Anopheles arabiensis and pyrethroidresistant Culex quinquefasciatus mosquitoes. Trans $R$ Soc Trop Med Hyg 2010, 104:639-645.

37. Foster WA, Takken W: Nectar-related vs. human-related volatiles: behavioural response and choice by female and male Anopheles gambiae (Diptera: Culicidae) between emergence and first feeding. Bull Entomol Res 2004, 94:145-157.

38. WHO: The Global Plan for Insecticide Resistance Management in Malaria Vectors (GPIRM). 2012.

39. President's Malaria Initiative: Malawi Malaria Operational Plan FY 2013. 2013.

40. Denholm I, Rowland MW: Tactics for managing pesticide resistance in arthropods: theory and practice. Annu Rev Entomol 1992, 37:91-112.

41. WHOPES: Report of the Sixteenth WHOPES Working Group Meeting WHO/HQ Geneva; Review of Pirimiphos-methyl 300CS, Chlorfenapyr 240 SC, Deltamethrin 62.5 SC-PE, Duranet LN, Netprotect LN, Yahe LN, Spinosad 83.3 Monolayer DT, Spinosad 25 Extended Release GR. 2013.

doi:10.1186/1475-2875-13-37

Cite this article as: Oxborough et al:: Long-lasting control of Anopheles arabiensis by a single spray application of micro-encapsulated pirimiphos-methyl (Actellic ${ }^{\otimes} 300$ CS). Malaria Journal 2014 13:37. 